\title{
Der Gemeinsame Bundesausschuss als Motor für die Initiierung klinischer Studien? Eine kritische
}

\section{Analyse}

\section{ANTJE GOTTBERG, \\ DIEDRICH BÜHLER, \\ BERNHARD EGGER}

Dr. Antje Gottberg ist Referentin für Grundsatzfragen in der Abteilung Medizin beim GKV-Spitzenverband, Berlin

Dr. Diedrich Bühler leitet das Referat Methodenbewertung beim GKV-Spitzenverband, Berlin

Dr. Bernhard Egger leitet die Abteilung Medizin beim GKV-Spitzenverband, Berlin

\author{
Die Rolle des Gemeinsamen Bundesausschusses bei der \\ Innovationsbewertung hat sich in den letzten Jahren \\ grundlegend gewandelt. Neben der Nutzenbewertung \\ auf Basis bereits vorhandenen Wissens hat der G-BA \\ zunehmend auch die Aufgabe übernommen, selbst Wissen \\ zu generieren und Studien zu initiieren. Aber kann das \\ wirklich gelingen? Aufbauend auf den Erfahrungen aus \\ einem frühen und prominenten Beispiel, der PREFERE- \\ Studie, wird diskutiert, welche förderlichen Faktoren \\ und welche Hindernisse für die Durchführung von \\ klinischen Studien zu nichtmedikamentösen Verfahren \\ in Deutschland bestehen. Anschließend werden \\ die aktuelle Gesetzgebung zu Erprobungsstudien \\ des G-BA bewertet und erste Vorschläge zu einer \\ Neugestaltung dieser Rahmenbedingungen präsentiert.
}

\section{Nutzenbewertung für ambulante und stationäre Methoden}

Ein Grundprinzip des deutschen Sozialrechts in Bezug auf die Verfügbarkeit von Untersuchungs- und Behandlungsmethoden für GKV-Versicherte ist die gegenläufige Wirkweise von „Erlaubnis- und Verbotsvorbehalt". Dabei gilt der Erlaubnisvorbehalt im vertragsärztlichen Bereich und der Verbotsvorbehalt im Krankenhaus. Das bedeutet, dass neue Methoden in der vertragsärztlichen Versorgung nur dann angewendet werden dürfen, wenn der Gemeinsame Bundesausschuss (GBA) dies nach einer Nutzenbewertung explizit erlaubt. Im Krankenhaus ist es umgekehrt: prinzipiell dürfen dort alle
Methoden zur Anwendung kommen, die der G-BA nicht ausdrücklich verboten hat. Dieses Grundprinzip wurde zwar in der jüngeren Vergangenheit durch diverse Gesetzesreformen modifiziert, war jedoch zum Beginn der Beratungen zu unserem folgenden Beispiel - der LDR-Brachytherapie - noch weitgehend uneingeschränkt maßgeblich.

\section{Beispiel Brachytherapie}

\section{- Die Grundlagen der Methode...}

Mit dem Beschluss zur Einführung der LDR-Brachytherapie zur Behandlung des Prostatakarzinoms endete am 17.09.2020 einer der längsten Beratungsprozesse des 
Gemeinsamen Bundesausschusses. Als Brachytherapie bezeichnet man die Bestrahlung von Tumorgewebe über eine kurze Distanz („,brachy“). Im vorliegenden Fall geht es um die so genannte „Low-dose-rate“ (LDR)-Brachytherapie der Prostata bei Männern mit Prostatakrebs. Hier werden etwa reiskorngroße radioaktiv beladene Strahlenquellen („Seeds“) in die Prostata eingebracht. Sie geben über einen bestimmten Zeitraum eine niedrig-dosierte („low dose“) Strahlung ab und verbleiben danach lebenslang im Organ. Im Vergleich zu einer operativen Entfernung der Prostata ist der Eingriff kurz und kann auch unter regionaler Anästhesie durchgeführt werden.

\section{...sind keineswegs neu}

Eine der Kernaufgaben des G-BA ist die Entscheidung über die Aufnahme neuer Untersuchungs- und Behandlungsmethoden in die ambulante Leistungspflicht der gesetzlichen Krankenversicherung. Auf dieser Grundlage stellte 2002 der Verband der Ersatzkassen einen Antrag auf Bewertung der LDR-Brachytherapie beim Prostatakarzinom beim Bundesausschuss der Ärzte und Krankenkassen, einem der Vorläufer des G-BA. Zu diesem Zeitpunkt gab es die Brachytherapie oder zumindest das eigentliche Behandlungsprinzip schon seit vielen Jahrzehnten. Die Methode als solche ist beinahe so alt wie die Entdeckung der Radioaktivität: bereits 1901, 5 Jahre nach Entdeckung des Radiums durch Marie Curie, fand das Element Verwendung zur Behandlung maligner Erkrankungen, sehr bald auch solcher der Prostata ${ }^{1}$. Die Entwicklung der Ultraschallbildgebung durch den Enddarm hat schließlich die heutige Applikationsform der Seeds ermöglicht, nämlich durch die Haut im Dammbereich. Spätestens seit den 1990er Jahren fand die Behandlungsart im Krankenhaus Anwendung bei der Behandlung von Prostatakarzinomen mit niedrigem Metastasierungsrisiko. Mit der Zunahme der Möglichkeiten zum ambulanten Operieren sollte sie den Patienten auch außerhalb der Krankenhausbehandlung zur Verfügung gestellt werden. Die niedergelassenen Ärzte konnten sie jedoch mangels Erlaubnis nicht zu Lasten der GKV erbringen, im vertragsärztlichen Sektor war die LDR-Brachytherapie also neu. Dies ist eine ganz typische Konstel- lation: Behandlungsmethoden werden im Krankenhaus schon in der Routineversorgung eingesetzt, eine Prüfung des Nutzens erfolgt jedoch erst, wenn die Methode auch ambulant angewandt werden soll.

\section{nutzenbewertung, 1. Runde}

Nach Annahme des Nutzenbewertungsantrags durch den Ausschuss erfolgte die Beauftragung des gerade neu gegründeten Instituts für Qualität und Wirtschaftlichkeit im Gesundheitswesen (IQWiG) im Dezember 2004. Bei einer Nutzenbewertung wird immer ein Vergleich der zu bewertenden Methode zu den in der GKV-Versorgung vorhandenen Alternativen durchgeführt. Beim lokal begrenzten Prostatakarzinom sind dies drei Behandlungsarten: die operative Entfernung der Prostata, die externe Strahlentherapie und die abwartende Beobachtung, bei der eine invasive Behandlung erst dann erfolgt, wenn der Tumor sein Ausbreitungsverhalten ändert. Zudem muss für die Bewertung vorab definiert werden, welche Zielgrößen („Endpunkte“) betrachtet werden sollen. In Frage kommen hier vorrangig so genannte patientenrelevante Endpunkte, also die Sterblichkeit (Mortalität), die Krankheitsschwere (Morbidität), die Lebensqualität und die Nebenwirkungen unter einer bestimmten Behandlung.

Wesentliche Voraussetzung für eine aussagefähige vergleichende Bewertung ist das Vorliegen von vergleichenden Studien. Als Goldstandard gelten hier randomisierte Studien, bei denen Patienten per Zufallszuteilung eine der untersuchten Behandlungsarten erhalten. Dies ist das beste Verfahren um sicherzustellen, dass gemessene Effekte tatsächlich durch die Interventionen selbst und nicht durch bekannte oder unbekannte Patienteneigenschaften hervorgerufen werden. Um es kurz zu machen: Studien der beschriebenen Art konnten nicht identifiziert werden. Letztendlich gingen zwar elf Studien in die Nutzenbewertung des IQWiG ein, keine von ihnen wies jedoch eine zufällige Patientenzuteilung auf und es wurden weitere Qualitätsmängel der Studien benannt. Es war andererseits kein Grund ersichtlich, warum RCTs nicht hätten durchgeführt werden können, da es sich um eine häufige Erkrankung handelt und es Therapiealternativen gibt. Der IQWiGAbschlussbericht endet mit der Feststellung, „Damit reichten die möglichen Vorteile der Brachytherapie hinsichtlich Organfunktion und Lebensqualität bei Patienten mit lokal begrenztem Prostatakarzinom als Nutzenbeleg allein für einen Einsatz dieses Therapieverfahrens nicht aus, da ein möglicher Schaden bezogen auf das Überleben und krankheitsbedingte Beschwerden nicht hinreichend sicher ausgeschlossen werden kann. Wir empfehlen dringend die Durchführung von aussagekräftigen klinischen Studien, um den Stellenwert der Brachytherapie im Vergleich zu den sonstigen Behandlungsoptionen zu definieren."2

\section{Nach dem IQWiG-Bericht 2007 stand der G-BA vor der Erkenntnis, dass die jahrzehntelange Anwendung der LDR-Brachytherapie nicht von der Schaffung einer belastbaren Datenbasis begleitet worden war.} 2007 stand der G-BA also vor der Erkenntnis, dass die jahrzehntelange Anwendung der LDR-Brachytherapie nicht von der Schaffung einer belastbaren $\mathrm{Da}$ tenbasis begleitet worden war, anhand derer die Anwender und Patienten sicher sein konnten, welche Vor- und Nachteile die Behandlung hat.

\section{Die PREFERE-Studie}

Im G-BA beschloss man, die Methode zunächst nicht in den ambulanten Leistungskatalog der GKV aufzunehmen, sondern die Beratungen auszusetzen und die vom IQWiG angemahnte Studie auf den Weg zu bringen. Der G-BA selbst

1 Zaorsky $\mathrm{N}$ et al. Evolution of brachytherapy for prostate cancer. Nat Rev Urol. 2017 June 30; 14(7): 415-439. doi:10.1038/ nrurol.2017.76.

2 IQWiG Abschlussbericht N04-02, https:// www.iqwig.de/download/N04-02_Abschlussbericht_Brachytherapie.pdf 
hatte damals noch keine gesetzliche Kompetenz, selbst Studien zu initiieren und durchzuführen. Die Aussetzung wurde an die Bedingung geknüpft, dass der GKV-SV ein Studienkonzept ${ }^{3}$ vorlegt, welches geeignet ist, den Nutzen der LDR-Brachytherapie im Vergleich $\mathrm{zu}$ den anderen Behandlungsmethoden nachzuweisen. In Zusammenarbeit mit dem IQWiG, dem Medizinischen Dienst des Spitzenverbandes (MDS), Vertretern einschlägiger Fachgesellschaf-

ten, Patientenorganisationen und der Deutschen Krebshilfe entstand das Konzept für eine komplexe randomisierte Studie. 7.600 Männer mit neu entdecktem Prostatakrebs sollten per Zufallszuteilung innerhalb von vier Jahren eine von vier Behandlungsarten bekommen: die operative Entfernung der Prostata, die externe Bestrahlung, die aktive Überwachung oder eben die LDRBrachytherapie. Aufgrund der sehr unterschiedlichen Therapiealternativen ging man davon aus, dass Männer in der Krankheitssituation die Behandlungsart kaum vollständig dem Zufall überlassen würden - anders, als wenn es beispielsweise um die Wahl zwischen zwei Medikamenten geht. Man hatte sich deswegen für ein „präferenzbasiertes“ Studiendesign entschieden; das bedeutet, dass ein Patient vor der Behandlungsauslosung bis zu zwei der Therapiearten „abwählen“ konnte, wenn sie für ihn überhaupt nicht in Frage kamen. Durch dieses komplexe Studiendesign gab es am Ende 11 Teilstudien.

Die Vielzahl betroffener Männer bei gleichzeitig unbefriedigender Datenlage über die Vor- und Nachteile der verschiedenen Behandlungsarten sprach für eine hohe Relevanz des Forschungsvorhabens: Immerhin wird diese Diagnose jährlich bei knapp 59.000 Männern gestellt ${ }^{4}$. Daher gelang es auch, die Finanzierung des Projekts sicherzustellen: Die Deutsche Krebshilfe hatte die Kostentragung für die der Forschungsleistungen zugesagt und außerdem die Entwicklung und Produktion evidenzbasierter Patientenaufklärungsmaterialien (Broschüren, Video) finanziert. GKV und Private Krankenversicherung (PKV) übernahmen u.a. die Kosten für die Patientenaufklärung und die Dokumentation sowie Schulung der beteiligten niedergelassenen Urologen. Darüber hinaus wurde die Vergütung der ambulant durchgeführten Brachytherapie im Rahmen der Studie durch einen kassenübergreifenden Vertrag zur integrierten Versorgung sichergestellt. Die Laufzeit der gesamten Studie war mit 14 Jahren geplant: 4 Jahre für die Rekrutierung der Patienten mit anschließend 10jähriger Nachbeobachtung. Zwar lief die Studie nach aufwändiger Öffentlichkeitsarbeit 2013 plangemäß an, letztendlich musste das ambitionierte Vorhaben

\section{Die Gründe für das Scheitern von PREFERE sind nie systematisch evaluiert worden, diverse Hürden sind im Vorfeld wohl unterschätzt worden.}

aber bereits 2016 beendet werden, weil es nicht ansatzweise gelungen war, die benötigte Anzahl von betroffenen Männern für die Teilnahme zu gewinnen. Für eine Teilnahme entschieden sich nur rund 340 Männer.

\section{Woran lag das Scheitern von PREFERE?}

Die Gründe für das Scheitern der Studie sind zwar nie systematisch evaluiert worden, diverse Hürden wurden aber genannt, die man im Vorfeld wohl auch unterschätzt hatte und trotz aufwändiger Begleitarbeit letztlich nicht abbauen konnte. Wichtigste Voraussetzung für das Gelingen war sicher, dass in Frage kommende Patienten überhaupt auf die Studie angesprochen wurden. Diese Aufgabe fiel in PREFERE den niedergelassenen Urologen zu, die in aller Regel das Prostatakarzinom diagnostizieren. Sie verlangt aber, dass jeder Urologe alle vier Therapieoptionen als gleichwertig darstellt, auch wenn er eigene Präferenzen hat ${ }^{5}$. Dies schien schwer vermittelbar angesichts der Tatsache, dass alle vier Verfahren einschließlich der LDR-Brachytherapie bereits seit Jahrzehnten zur Anwendung gekommen waren.

Hinzu kamen strukturelle Gründe: im Versorgungsalltag haben niedergelassene Ärztinnen und Ärzte meist ein Netzwerk von bestimmten Kliniken, die sie ihren Patienten für Operationen oder andere Behandlungen empfehlen. Allerdings waren nur vergleichsweise wenige Kliniken in Deutschland auch Prüfzen- tren der PREFERE-Studie, so dass die Praxen ihre Überweisungsgewohnheiten hätten ändern müssen. Nur wenige Zentren konnten diese Hürde durch aktive Netzwerkarbeit überwinden.

Dennoch sind RCT auch in diesem Umfeld möglich. Immerhin liegt solch hochwertige Evidenz zumindest für die Behandlung durch Operation, externe Bestrahlung und aktive Überwachung vor. Exemplarisch seien hier eine Studie aus Skandinavien ${ }^{6}$ und die PIVOTStudie $^{7}$ genannt. Sie wurden bereits in den frühen 90er Jahren des vergangenen Jahrhunderts begonnen und verfügen über dementsprechend lange Nachbeobachtungszeiten und Daten zum Langzeitüberleben. Allerdings dauerte der Einschluss von jeweils rund 700 Patienten auch 8-10 Jahre. In der randomisierten ProtecT-Studie $^{8}$ (n = 1643) wurden schließlich als primäre Therapieoptionen aktive Überwachung, radikale Prostatektomie und Strahlentherapie verglichen.

\section{Nutzenbewertung, 2. Runde}

Mit dem Abbruch der PREFERE-Studie entfiel für den G-BA auch die Grundlage für die Aussetzung der Beratungen. Das IQWiG wurde 2017 mit einer erneuten Recherche beauftragt um zu prüfen, ob sich seit der letzten Recherche im Jahr 2010 die Datenbasis zur Behandlungsmethode verändert hatte. Die Datenlage wurde auch 12 Jahre nach der ersten Recherche weiterhin als wenig aussagekräftig bewertet, da auch international keine aussagekräftigen RCTs zur Brachytherapie abgeschlossen worden sind. Im Ergebnis ist eine Nutzen-Schaden Abwägung der Brachytherapie aufgrund fehlender

3 Registereintrag unter ClinicalTrials.gov Identifier: NCT01717677

4 https://www.rki.de/DE/Content/Service/ Presse/Pressemitteilungen/2019/16_2019. html

5 Albers P. PREFERE-STUDIE (2) - Was ist für die Zukunft abzuleiten? Deutsches Ärzteblatt | Jg. 113 | Heft 50 | 16. Dezember 2016, A2314-16

6 Bill-Axelson A, Holmberg L, Ruutu M, et al.; SPCG-4 Investigators. Radical prostatectomy versus watchful waiting in early prostate cancer. N Engl J Med 2011; 364: 1708-17.

7 Wilt TJ, Brawer MK, Jones KM, et al.; Prostate Cancer Intervention versus Observation Trial (PIVOT) Study Group. Radical prostatectomy versus observation for localized prostate cancer. N Engl J Med 2012; 367: 203-13.

8 Hamdy, F.C., et al., 10-Year Outcomes after Monitoring, Surgery, or Radiotherapy for Localized Prostate Cancer. N Engl J Med, 2016. 375(15): p. 1415-1424. 
Daten - trotz jahrzehntelanger Anwendung - weiterhin nicht möglich. Daher ist der Nutzen der LDR-Brachytherapie im Vergleich zu allen anderen Behandlungsoptionen im Hinblick auf das Behandlungsziel „Heilung" nach wie vor unklar.

Mehrere abgebrochene RCTs in England, Kanada und den USA $^{9}$ verdeutlichen die Herausforderungen der Forschung auf diesem Feld: Das Prostatakarzinom ist eine Erkrankung, die (glücklicherweise) sehr langsam voranschreitet. Das bedeutet, dass man sehr große Gruppen von erkrankten Männern über sehr viele Jahre beobachten muss, um aussagefähige $\mathrm{Da}$ ten zur Überlebenswahrscheinlichkeit unter den verschiedenen Behandlungsarten zu erhalten. Schneller hingegen erhält man verwertbare Daten zu Therapienebenwirkungen, die ja meist unmittelbar oder zumindest in einem überschaubaren Zeitraum nach den Interventionen eintreten. Die Verschiedenheit der Therapieoptionen macht es zusätzlich sehr schwierig, Männer davon zu überzeugen, die Wahl der Behandlung einem Zufallsverfahren zu überlassen. Selbst ein präferenzbasiertes Studiendesign wie in der PREFERE-Studie, in dem die Patienten Einfluss auf die möglichen Interventionen nehmen konnten, erhöhte die Bereitschaft zur Teilnahme nicht wesentlich.

- Abschließende Beratungen im G-BA

Vor dem Hintergrund einer absehbar nicht mehr verbesserungsfähigen Evidenzlage musste der G-BA letztlich eine Entscheidung treffen. Die Leistung wurde eingeführt, explizit begrenzt auf die Durchführung bei Tumoren der niedrigsten Risikokategorie. Überdies sollen begleitende Maßnahmen der Sicherung der Behandlungsqualität dienen. Eine dieser Maßnahmen ist die bestmögliche Förderung der informierten Entscheidung des Patienten. Das IQWiG wurde daher beauftragt, eine evidenzbasierte Entscheidungshilfe zu entwickeln. Sie stellt dar, mit welcher Sicherheit Aussagen zur onkologischen Wirksamkeit für die jeweiligen Therapiearten getroffen werden können und erläutert die Datenlage zu den unterschiedlichen Nebenwirkungsprofilen. Die Richtlinie zu den Qualitätssicherungsmaßnahmen sieht die Verpflichtung vor, jedem Patienten im Zuge der vorbereitenden Gespräche dieses Dokument auszuhändigen. So hat der G-BA eines seiner längsten Beratungsverfahren beendet. Die Eckdaten des Verfahrens sind in Abbildung 1 dargestellt.

Bei der Entscheidung zur LDR-Brachytherapie der Prostata spielen letztlich spezifische medizinische Eigenheiten der Erkrankung und der Therapiemethoden eine wesentliche Rolle. Es bleibt die Frage, wie dieser Beschluss des G-BA zu bewerten ist und welche Folgen sich dabei für andere Beratungsverfahren ergeben. Wird der G-BA insgesamt seiner Verantwortung gerecht, dafür zu sorgen, dass der Nutzen von Methoden verlässlich geklärt wird, bevor sie in der breiten Versorgung angewandt werden?

\section{Durchführung von klinischen Studien im G-BA: die Zeit nach der Brachytherapie}

Im Vorfeld der PREFERE-Studie konnte der G-BA nach geltendem Recht nur seine Beratungen aussetzen und abwarten. Der GKV-SV übernahm die Aufgabe, eine konkrete Studie zu planen. Mit dem GKV-Versorgungsstrukturgesetz, das am 1.1.2012 in Kraft trat, kann der
G-BA nun selbst Studien initiieren und beauftragen. Ist das aber eine Garantie dafür, dass diese Studien auch gelingen? Leider nein. Denn häufig werden kritische Abschlussberichte des IQWiG von den Krankenhausvertretern und den wissenschaftlichen Fachgesellschaften grundlegend in Frage gestellt, wenn es sich um eine „neue“ Methode für den ambulanten Sektor handelt, die im Krankenhaus bereits breit angewendet wird. Vorliegende Studien werden dann sehr unterschiedlich interpretiert und häufig wird die faktische Möglichkeit verneint, die offenen Fragen durch eine klinische Studie zu klären. Aktuelles Beispiel hierfür ist die Positronen-Emissions-Tomografie (PET), ein bildgebendes diagnostisches Verfahren, das vor allem in Onkologie angewandt wird. $\mathrm{Zu}$ vielen Einsatzgebieten der PET wurden mit Beschluss des G-BA vom 20.11.2020 die Beratungen eingestellt: Eine Nutzenanerkennung war nicht möglich. Die Durchführung von Studien gelang aber auch nicht. Damit musst der G-BA auf die Tatsache reagieren, dass auch ohne Anerkennung der Leistung durch den Gemeinsamen Bundesausschuss die Versorgungsrealität ohnehin bereits Fakten geschaffen hat. PET-Untersuchungen werden in großen

9 Crook JM, Gomez-Iturriaga A, Wallace K, Ma C, Fung S, Alibhai S et al. Comparison of health-related quality of life 5 years after SPIRIT: surgical prostatectomy versus interstitial radiation intervention trial. J Clin Oncol 2011; 29(4): 362-368. 
Umfang entweder im Rahmen von Einzelfallentscheidungen der Krankenkassen, von Selektivverträgen oder im Rahmen der ambulant spezialfachärztlichen Versorgung (ASV) erstattet. Das verweist auch auf die Notwendigkeit, mit versorgungsregulierenden Maßnahmen den richtigen Zeitpunkt zu treffen.

\section{Was sind derzeit die positiven und negativen Erfolgsfaktoren für G-BA-initiierte Studien?}

Während frühere Forschungsvorhaben in Folge von Beratungen im G-BA (siehe PREFERE, aber auch zur Vakuumversiegelungstherapie von Wunden) auf Initiativen außerhalb des G-BA angewiesen waren, steht mit dem Inkrafttreten des $\$ 137$ e SGB V im Jahr 2012 jetzt ein Regelwerk zur Verfügung, auf dessen Basis der G-BA für Untersuchungs- und Behandlungsmethoden mit unzureichendem Nutzenbeleg Erprobungsstudien auflegen kann bzw. muss. Hierzu beschließt der G-BA jeweils eine Richtlinie zur Erprobung, in der die Eckdaten einer Studie festgelegt sind, deren Ergebnisse zur späteren Nutzenbewertung dienen sollen. Dabei herrscht Konsens, dass es sich in der Regel um randomisierte, kontrollierte Studien handeln muss. Eine Übersicht über alle Erprobungsvorhaben findet sich unter https://www.g-ba.de/studien/. Bislang ist noch keine dieser Studien abgeschlossen. Aus der Projektbegleitung gibt es aber Hinweise darauf, welche Faktoren zum Gelingen beitragen können. In erster Linie sind dies natürlich zunächst die Faktoren, die für jedes klinische Forschungsvorhaben maßgeblich sind:

\section{- Der Zeithorizont}

Grundsätzlich ist eine Studie leichter durchführbar, wenn ihr Zeithorizont und die Zahl der einzuschließenden Probanden überschaubar sind: Vor diesem Hintergrund stellt beispielsweise die Erprobung der allogenen Stammzelltransplantation beim multiplen Myelom mit einer geplanten Laufzeit von mindestens 10 Jahren unter Einschluss von 400 schwerstkranken Patienten mit Sicherheit eine große Herausforderung dar. Dem gegenüber erscheint die Erprobung von zwei unterschiedlichen Verfahren zur Mandelentfernung vergleichsweise unkompliziert: die Erkrankung ist häufig, es werden viele Patienten operiert und die Nachbeobachtungszeit beträgt zwei Jahre.

\section{- Die Attraktivität (für Versicherte und Leistungserbringer)}

Ein mit Sicherheit maßgeblicher Faktor ist aber auch, dass die Studienteilnahme für Patientinnen und Patienten attraktiv ist. Dies kann dann der Fall sein, wenn sie in der Studie eine Behandlung bekommen, die ihnen ansonsten, zumindest im Rahmen der GKV-Versorgung, nicht zur Verfügung steht. Als Beispiel sei hier die Liposuktion (Fettabsaugung) beim Lipödem genannt, einer schmerzhaften Fettverteilungsstörung. Bislang müssen die betroffenen Frauen die Operationen privat bezahlen, oft fünfstellige Summen. Im Rahmen der Studie bezahlen die Krankenkassen die Behandlung, wie es das Gesetz vorsieht. In einer Datenbank sind vor Studienbeginn erheblich mehr interessierte Frauen registriert, als für die Studie benötigt werden, so dass ein Losverfahren zum Einsatz kommen muss. Die am Beispiel der Brachytherapie beschriebenen Rekrutierungsprobleme sind hier also offensichtlich nicht relevant. Auch bei Erkrankungen mit schlechter Prognose, für die bislang keine wirksamen Behandlungen zur Verfügung stehen, kann eine Studienteilnahme attraktiv sein, wenn sich daraus zumindest die Hoffnung auf eine Besserung oder Verlangsamung des Krankheitsfortschritts speist. Dieser Faktor könnte bei der Erprobung einer neuen Behandlung der Retinopathie, einer zur Erblindung führenden Netzhauterkrankung, zum Tragen kommen.

\section{- Die Verfügbarkeit der Anwendung außerhalb der Studie}

Umgekehrt lässt sich daraus aber auch ableiten: Ist die Behandlung GKV-Versicherten bereits zugänglich, so werden diese sich kaum einer Randomisierung unterziehen mit dem „Risiko“, eine präferierte Behandlung nicht zu erhalten. Ein ganz wesentlicher Faktor in diesem Sinne ist der Verbotsvorbehalt im Krankenhaus. Wenn die Leistung stationär bereits breit zur Verfügung steht, ist schwer zu vermitteln, dass vor Übernahme in den ambulanten Leistungskatalog eine klinische Studie durchgeführt werden soll. Dies führt in der Wahrnehmung von Vielen dazu, dass das Erfordernis einer Nutzenbewertung und der Durchführung klinischer Studien dann als eher bürokratisches Hindernis wahrgenommen wird. Denn im Krankenhaus geht es ja auch ohne. In den letzten Jahren sind noch weitere solcher Systembrüche hinzugekommen bzw. haben an Bedeutung gewonnen. So gilt für Hochschulambulanzen und seit 2012 auch für die ambulante spezialfachärztliche Versorgung der Verbotsvorbehalt. Zudem können Krankenkassen mit einzelnen Leistungsvertretern Selektivverträge abschließen, innerhalb derer Behandlungsarten angewendet werden, die der G-BA nicht explizit ausgeschlossen, in der Regel also noch gar nicht beraten hat. Kurz: Bei Behandlungen, die bereits in der Versorgung etabliert sind, sei es durch lange Anwendung im Kranken-

\section{Bei Behandlungen, die bereits in der Versorgung etabliert sind, lässt sich kaum überzeugend darstellen, dass hier noch Forschungsbedarf besteht.}

haus oder bestehende Selektivverträge, lässt sich kaum überzeugend darstellen, dass hier noch Forschungsbedarf besteht und Betroffene sich der Mühsal und den Unwägbarkeiten einer klinischen Studie unterziehen sollen. Die Generierung weiterer Evidenz, so notwendig dies aus wissenschaftlicher Sicht auch sein mag, wird dann in der Tat faktisch unmöglich.

\section{- Marktzugang}

Demgegenüber kann das Interesse von Medizinprodukteherstellern eine Studie deutlich befördern. Es besteht hier doch die Chance, Marktzugangsstudien auf Kosten des Beitragszahlers durchzuführen. Dies mag ein Grund dafür gewesen sein, dass eine frühere Studie des G-BA erfolgreich zu Ende geführt wurde: Die Ergebnisse der randomisierten Studie zur Vakuumversiegelungstherapie von Wunden führten dazu, dass im Jahr 2019 ein ebenfalls sehr langes Beratungsverfahren abgeschlossen werden konnte. Als Ergebnis kann diese Behandlung nunmehr auch in der ambulanten Versorgung zum Einsatz kommen. 


\section{- Strukturkompatibilität}

Schlussendlich ist es vorteilhaft, wenn die Rahmenbedingungen für die Durchführung einer Studie weder von der alltäglichen klinischen Versorgungsrealität noch von den üblichen Routinen der klinischen Forschung allzu sehr abweichen. Förderlich wäre demnach, wenn zwischen Studienzentren und weiteren beteiligten Leistungserbringern bereits in der Regelversorgung bewährte Netzwerke bestünden. Auch wenn es in einigen Umsetzungsfragen noch Nachbesserungsbedarf gibt, ist die Finanzierung über GKV-Mittel grundsätzlich umfassend gesichert, sowohl was die Kosten für die medizinischen Leistungen als auch des wissenschaftlichen Overheads angeht.

\section{Die aktuelle Gesetzgebung zu Erprobungsstudien des G-BA: hinderlich, förderlich oder nur ein Alibi?}

Der Gesetzgeber hat dem G-BA in den letzten Jahren zunehmend mehr Kompetenzen aber auch mehr Verantwortung bei der Durchführung klinischer Studien übertragen. Die Gesetzgebung fußt offensichtlich zum großen Teil auf der Hypothese, dass die Finanzierung der Studien das entscheidende Hindernis für die praktische Umsetzung darstellt. Derzeit trägt der G-BA - und damit der Beitragszahler der GKV - die volle finanzielle Last der Kosten der wissenschaftlichen Durchführung (wissenschaftlicher Overhead) aller Erprobungsstudien, sofern nicht ein Hersteller selbst und freiwillig erklärt, dass er die Studie auf eigene Kosten durchführen möchte. Die Kosten für die medizinischen Leistungen im Rahmen der Studien tragen die Krankenkassen. Das sind Rahmenbedingungen, die international gesehen einmalig sind, aus Sicht der GKV aber falsche Anreize setzen und für die Methodenbewertung im G-BA erhebliche Auswirkungen haben.

Im Krankenhausbereich ist der Ausschluss von Leistungen ohne nachge- wiesenen Nutzen durch die neuere Gesetzgebung kaum mehr durchzusetzen. Leistungsausschlüsse bedürfen eines 9-Stimmen-Quorums im Plenum und können nur noch dann erfolgen, wenn eine Methode kein „Potenzial einer erforderlichen Behandlungsalternative" besitzt. Der Nutzen muss nicht belegt sein, es reicht eine (allerdings durch empirische Daten zu belegende) begründete Erwartung, dass die Methode „besser“ sein könnte. Der G-BA führt, wenn er dieses feststellt, für solche Methoden mit „Potenzial“ dann eine Erprobungsstudie auf Kosten des Beitragszahlers durch. In der Regel gibt es aber keinerlei Interesse bei den Krankenhäusern, eine solche Studie auch wirklich durchzuführen, da die Leistung auch außerhalb einer solchen Erprobungsstudie von der GKV finanziert werden muss. Ähnlich ist die Situation bei der ursprünglich mit großen Hoffnungen verbundenen Gesetzgebung aus dem Jahre 2015 zu den Hochrisikomedizinprodukten in $\$ 137 \mathrm{~h}$ SGB V. Damit wurden die Krankenhäuser eigentlich verpflichtet, sich an klinischen Studien zu beteiligen, wenn sie bestimmte neue Methoden unter Einsatz von Hochrisikomedizinprodukten anwenden möchten. Die Bilanz war hier bislang sehr ernüchternd. Bisher wurde keine einzige Studie auf dieser Rechtsgrundlage initiiert. Nunmehr wurde der Zwang zu Beteiligung an Studien für die anwendenden Krankenhäuser ganz explizit aufgehoben. Deswegen und Aufgrund der Finanzierungsanreize des Verbotsvorbehalts im stationären Sektor ist fraglich, ob den nächsten Jahren wirklich auf diesem Wege relevante Erkenntnisse generiert werden können. Vielmehr schafft die sogenannte „Erprobung“ eher den Rechtsrahmen für die Anwendung außerhalb eines RCT.

Anders ist die Situation (noch) im Bereich der vertragsärztlichen Versorgung. Hier gilt weiterhin, dass eine neue Leistung ohne Nutzenanerkennung durch den G-BA nicht in die Leistungspflicht der GKV fällt. Das Gesetz sieht seit Dezember 2019 zudem vor, dass der G-BA in Folge von Methodenbewertungsverfahren im ambulanten Bereich praktisch immer eine Erprobungsstudie durchführen muss, wenn keine Aufnahme der entsprechenden Leistung erfolgt, die Innovation aber das oben bereits erwähnte „Potenzial einer erforderlichen Behandlungsalternative" besitzt. Hersteller von Medizinprodukten und Leistungserbringer können aufgrund einer gesetzlichen Neuregelung vom Dezember 2011 gemäß \$137e SGB V auch selbst Erprobungen beantragen. Bis Ende 2019 galt, dass sich die Hersteller an den Kosten für den wissenschaftlichen Overhead „angemessen“ beteiligen mussten. Das mündete in langwierige und meist ergebnislose Klärungsprozesse mit den Antragstellern, was denn „angemessen" wäre und ob der Antragsteller

\begin{tabular}{|c|c|c|c|}
\hline Erprobungs-Richtlinie & $\begin{array}{l}\text { Patientenzahl } \\
\text { (geplant) }\end{array}$ & $\begin{array}{l}\text { Laufzeit } \\
\text { (Jahre) }\end{array}$ & Status \\
\hline $\begin{array}{l}\text { Magnetresonanztomographiegesteuerte hochfokussierte } \\
\text { Ultraschalltherapie zur Behandlung des Uterusmyoms }\end{array}$ & 127 & 4 & gestartet \\
\hline $\begin{array}{l}\text { Transkorneale Elektrostimulation bei Retinopathia } \\
\text { Pigmentosa }\end{array}$ & 134 & 5,5 & In Vorbereitung \\
\hline $\begin{array}{l}\text { Messung und Monitoring des pulmonalarteriellen Drucks } \\
\text { bei Herzinsuffizienz }\end{array}$ & 554 & 4 & gestartet \\
\hline Stammzelltransplantation bei Multiplem Myelom & 400 & $10-12$ & In Vorbereitung \\
\hline Liposuktion zur Behandlung des Lipödems & 450 & 5 & In Vorbereitung \\
\hline $\begin{array}{l}\text { Tonsillotomie zur Behandlung der rezidivierenden akuten } \\
\text { Tonsillitis }\end{array}$ & 454 & 5 & gestartet \\
\hline $\begin{array}{l}\text { Pulsierende elektromagnetische Felder bei Knochenbruch- } \\
\text { heilungsstörungen der langen Röhrenknochen }\end{array}$ & $\begin{array}{l}\text { Schätzung: } \\
200-400\end{array}$ & & Vergabe läuft \\
\hline $\begin{array}{l}\text { Aktive Kniebewegungsschienen zur Selbstanwendung } \\
\text { bei Rupturen des vorderen Kreuzbands }\end{array}$ & 90 & 3 & In Vorbereitung \\
\hline Amyloid-PET bei Demenz unklarer Átiologie & $\begin{array}{l}\text { Schätzung: } \\
1000-1300\end{array}$ & & Vergabe läuft \\
\hline
\end{tabular}

Quelle: Eigene Darstellung 
diese Kosten aufbringen will und kann. Der Gesetzgeber hat darauf mit einem weiteren Schritt im Sinne der Verlagerung der Finanzierungsverantwortung auf den Betragszahler reagiert. Seit diesem Jahr muss der G-BA sämtliche wissenschaftlichen Studienkosten auch für von Herstellern beantragte Erprobungsstudien übernehmen, es sei denn, der Hersteller erklärt sich bereit, die Kosten selbst zu tragen. Bei Erfüllung bestimmter inhaltlicher und formaler Voraussetzungen muss der G-BA demnach auf Antrag von Medizinprodukteherstellern oder Leistungserbringern auf Kosten der Beitragszahler klinische Studien durchführen. Inzwischen sind einige Studien konkret gestartet und haben zum Teil bereits Patientinnen und Patienten eingeschlossen (Abbildung 2).

Dabei fällt auf, dass es sich vielfach um primär technisch und vom Einsatz teurer Medizinprodukte geprägte Verfahren handelt. Eine Ausnahme ist hier bestenfalls die Liposuktion, die primär durch eine Patienteninitiative auf den Weg gebracht wurde. Was bisher überhaupt keine Rolle spielt, sind nicht-technische Innovationen z.B. aus dem Bereich der zuwendungsorientierten Versorgung, komplexer Interventionen oder der Psychotherapie. Genutzt wird die Studienregelung beim G-BA, wie wohl auch gesetzgeberisch intendiert, bisher hauptsächlich von der Medizinprodukteindustrie. So werden künftig wohl deutlich mehr Versichertengelder auch für Studien ausgegeben, deren Versorgungsrelevanz nicht immer klar ist. Im schlimmsten Fall werden sie dazu genutzt, teure technische Innovationen mit primärem Herstellerinteresse klinisch zu erproben.

\section{Was sollte sich ändern?}

Aus unserer Sicht sollten die Rahmenbedingungen für die Durchführung klinischer Studien durch den G-BA vor dem Hintergrund der Erfahrungen der letzten Jahre neugestaltet werden. Kernpunkt ist, dass Innovationen in einem möglichst frühen Stadium nach verbindlichen Vorgaben in einer klinischen
Studie untersucht werden müssen. Das wird in der Regel nur gelingen, wenn die Anwendung und Finanzierung der Innovation beschränkt ist auf die Studie selbst. Die Rahmenbedingungen im stationären Sektor sehen durchweg ein Nebeneinander von klinischer Studie und Anwendung in der Fläche vor. Damit ist unklar, wer am Ende (außer dem mit der Bewertung der Methode beauftragten G-BA) ein echtes Interesse am Gelingen dieser Studien haben soll. Ob solche Studien im G-BA-Kontext trotzdem gelingen können, erscheint fraglich. Die wirtschaftlichen Anreizstrukturen sind dafür eher ungünstig. Völlig unproduktiv ist es, den G-BA zur Durchführung von Studien zu zwingen, die mit großer Wahrscheinlichkeit nicht gelingen können oder keine relevanten Konsequenzen in der Versorgungsrealität haben werden. Solche Studien - sollten sie jemals zustande kommen - dienen im Kontext der aktuellen Gesetzgebung zu den Hochrisikomedizinprodukten unserer Ansicht nach hauptsächlich als Alibi dafür, dass technische Innovationen bereits in einem frühen und unausgereiften Stadium in der Fläche zu Lasten des Beitragszahlers eingesetzt werden können.

Anders sieht dies im vertragsärztlichen Bereich aus. Auch hier werden in neuerer Zeit Aufweichungstendenzen des Grundprinzips, dass erst der Nutzen belegt werden muss, bevor eine Leistung bezahlt wird, wirksam (ambulante spezialfachärztliche Versorgung, Hochschulambulanzen, digitale Gesundheitsanwendungen, Selektivverträge der Krankenkassen). Überwiegend ist dieses Grundprinzip aber noch intakt, so dass echte wirtschaftliche Anreize für eine Durchführung aussagekräftiger klinischer Studien bestehen. Eigentlich ist die Finanzierung solcher Studien nicht Aufgabe der GKV. Hier hat der Gesetzgeber aber anders entschieden. Aus unserer Sicht sollte dann aber zumindest sichergestellt werden, dass die Versichertengelder nur in Studien investiert werden, die voraussichtlich erfolgreich durchgeführt werden können für die es einen echten Bedarf in der Versorgung gibt.

\section{Fokus Kindergesundheit}

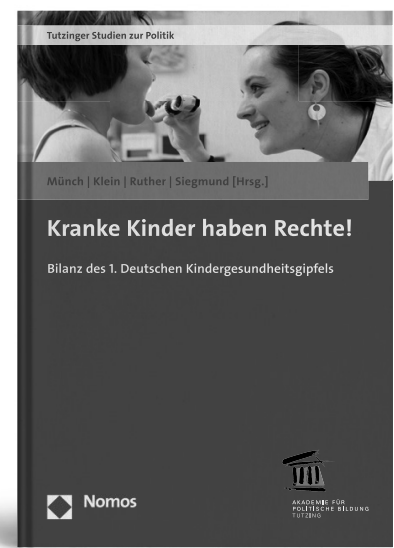

Kranke Kinder haben Rechte!

Bilanz des 1. Deutschen Kindergesundheitsgipfels Herausgegeben von Prof. Dr. Ursula Münch, Prof. Dr. Dr. Christoph Klein, Dr. Carolin Ruther und Jörg Siegmund

2021, ca. 250 S., brosch., ca. 49,- $€$ ISBN 978-3-8487-7791-4

(Tutzinger Studien zur Politik, Bd. 19) Erscheint ca. März 2021

In einem Gesundheitssystem, das zunehmend auf Effizienz und Optimierung ausgerichtet ist, werden die Bedürfnisse und Rechte kranker Kinder oft übergangen. Der Band dokumentiert aus einer interdisziplinären Perspektive die Beiträge des 1. Deutschen Kindergesundheitsgipfels zu den Herausforderungen der Kindermedizin.

\section{三. eLibrary}

X Nomos 